effects of vitamin $E$ induced neurologic dysfunction. In addition, electrophysiological studies may be a good variable to measure the effects of treatment.

This research was supported by the Lori and Bernard Levmore Research Fund.

\footnotetext{
References

1 Johanson A, Blizzard R. A syndrome of congenital aplasia of the alae nasi, deafness, hypothyroidism, dwarfism, absent permanent teeth and malabsorption. $J$ Pediatr 1971;79:982-7.

2 Townes PL, White MR. Identity of two syndromes. Proteolytic lipolytic and amylocytic deficiency of the exocrine pancreas with congenital anomalies. Am J Dis Child 1981;135:248-50.

${ }^{3}$ Farrell P, Bieri JG, Fretantoni JF, Wood RE, di Sant Agnese P.
}

The occurrence and effects of human vitamin E deficiency. J Clin Invest 1977;60:233-41.

${ }^{4}$ Bye AME, Muller DPR, Wilson J, Wright VH, Mearns MB. Symptomatic vitamin E deficiency in cystic fibrosis. Arch Dis Child 1985;60:162-4.

5 Muller DPR, Lloyd JK, Bird AC. Long term management of A- $\beta$-lipoproteinemia: possible role for vitamin E. Arch Dis Child 1977;52:209-14

${ }^{6}$ Harding AE, Muller DPR, Thomas PK, Willison HJ. Spinocerrebellar degeneration secondary to chronic intestinal malabsorption: a vitamin E deficiency syndrome. Ann Neurol 1982;12:419-24.

Correspondence to Dr S Freier, Shaare Zedek Hospital, Jerusalem, Israel.

Received 8 May 1986

\title{
Recurrent sepsis and gastrointestinal ulceration due to child abuse
}

\author{
L G RUBIN, A ANGELIDES, M DAVIDSON, AND P LANZKOWSKY
}

Department of Pediatrics, Schneider Children's Hospital of Long Island Jewish Medical Center and Health Sciences Center, State University of New York at Stony Brook, United States of America

SUMMARY An 11 year old girl developed progressive upper gastrointestinal ulceration and recurrent episodes of intravenous catheter associated polymicrobial septicaemia. Evaluation failed to establish a cause. After exclusion of the parents and careful surveillance of the patient she improved, supporting the diagnosis of suspected child abuse (a form of Meadow's syndrome).

Meadow's syndrome (Munchausen's syndrome by proxy) is a form of child abuse in which a parent feigns illness in his or her child. ${ }^{1}$ In a variant form the parent actually induces illness in the child. The diagnosis is confirmed by surreptitious observations ${ }^{2}$ confrontation of the parents and admission of guilt, ${ }^{3}$ and/or selected laboratory tests. ${ }^{4}$ This paper reports a girl with recurrent septicaemia and gastrointestinal ulceration of unknown aetiology who progressively deteriorated over a period of one year. She improved after exclusion of her parents and constant surveillance.

\section{Case report}

An 11 year old girl weighing $39 \mathrm{~kg}$ developed fever, cough, and protracted vomiting in January 1983, two months after a gastric ulcer had been diagnosed in her 14 year old brother. During a five week admission to hospital she had persistent vomiting and intermittent diarrhoea. She was transferred to Schneider Children's Hospital where examination revealed a thin, mildly dehydrated child weighing $33.8 \mathrm{~kg}$ who was otherwise normal.

Laboratory evaluation showed a haemoglobin concentration of $12.6 \mathrm{~g} / \mathrm{dl}$ and serum protein concentration of $5.9 \mathrm{~g} / 100 \mathrm{ml}$. The following tests yielded normal or negative results: leucocyte count and differential, erythrocyte sedimentation rate, serum albumin, serum electrolyte, and immunoglobulin concentrations, urinalysis, stool culture, three stool examinations for ova and parasites, chest radiography, and barium contrast studies of the upper and lower gastrointestinal tracts, including views of the terminal ileum. Upper gastrointestinal endoscopy revealed a solitary lesser curve gastric ulcer. Cimetidine and antacids were administered with satisfactory clinical response.

Five days after discharge she developed a recurrence of abdominal pain and intractable vomiting and complained of a sore throat. On re-admission physical examination was remarkable for an erythematous pharynx and a tongue ulcer. Repeat endoscopic examination 20 days after the first revealed persistence of the gastric ulcer and a new deep $2 \mathrm{~cm}$ ulcer of the distal oesophagus. Serum amylase and gastrin activities were normal as was a secretin stimulation study. Sucralfate was added to the regimen and the cimetidine increased. After discharge episodes of vomiting and abdominal pain 
recurred and in May 1983 a mid-oesophageal stricture was documented. Oesophageal dilatation was performed but continued dysphagia necessitated a fundoplication and gastrostomy in June. Abdominal exploration at that time revealed no evidence of inflammatory disease involving the small or large bowel. Liver, gall bladder, biliary tree, and pancreas were normal. In September 1983 the patient developed severe abdominal pain and intolerance to feedings after she was given an antibiotic (ampicillin) through the gastrostomy tube. Endoscopic examination revealed multiple ulcerations of the gastric antrum, immediately distal to the gastrostomy tube site. Subsequently, feeding intolerance recurred, requiring re-admission for treatment of dehydration on two occasions during the next two months.

During the patient's admission to hospital in May 1983 after diagnosis of the oesophageal stricture, total parenteral nutrition was begun through a centrally placed catheter. There was an acute onset of fever associated with visible particulate material in the alimentation bottle. Blood culture yielded negative results, but fluid from the bottle grew Candida albicans, Staphylococcus epidermidis, and Streptococcus viridans. Culture of hyperalimentation fluid (routinely performed by the hospital pharmacy before dispensing of the fluid) yielded negative results. Furthermore, no other patients with abnormal or infected hyperalimentation fluid were noted at the time.

Over the next six months there was a recurrent pattern of abrupt fever associated with bacteraemia; all these episodes occurred at a time when an intravascular catheter was in place. She had 39 positive blood cultures over eight months, which grew two fungal and at least 15 different bacterial species. They were often polymicrobial (12 blood cultures), caused by micro-organisms that are unusual in catheter associated bacteraemia-for example, Veillonella parvula -and often due to organisms that are normal oral or faecal flora. Furthermore, these episodes were often associated with purulent drainage and phlebitis at the catheter site, and there was a prompt response when specific antimicrobial treatment was combined with removal of the catheter. Immunologic evaluation repeatedly showed normal numbers of polymorphonuclear leucocytes, lymphocytes, and monocytes. A nitro blue tetrazolium test, and tests of immunoglobulins, complement $(\mathrm{C} 3, \mathrm{C} 4$, and $\mathrm{CH} 50)$, and cellular immunity yielded normal results. A gallium scan, computed tomograms of head and abdomen, and multiple echocardiograms as well as abdominal exploration and thoractomy with mediastinal and oesophageal exploration failed to reveal any abscess, focus of infection, or source for continuous seeding of the bloodstream.

Throughout the patient's multiple and prolonged admissions to hospital the mother was constantly at the bedside. Rather than appearing discouraged by her daughter's continued deterioration, she provided the nursing and medical staff with constant encouragement. She applauded the staff for their efforts and assured them they were doing everything possible. Though the parents were encouraged to seek evaluation at another tertiary care centre for further opinion and management, they returned to Schneider Children's Hospital, where the mother and the child felt 'more comfortable'. In October 1983 the mother was confronted with the suspicion that she was instrumental in the production of the bacteraemias. Her response was 'I can see how you might think that', but she denied contaminating the indwelling catheters.

The final admission of the child to our hospital was in late December 1983. Several days after transfer, abdominal exploration revealed a perforated jejunum (after passage of a transpyloric feeding tube at the local hospital). Careful abdominal exploration during laparotomy showed no evidence of chronic inflammatory bowel disease. One week later she developed high fever, became hypotensive, and was critically ill. Multiple blood cultures became positive for Candida albicans, and amphotericin B was administered. She improved, but 10 days later Streptococcus faecalis bacteraemia was associated with thrombosis and infection at a femoral catheter site. Chest pain and pulmonary infiltrates developed and lung scan findings were consistent with the clinical diagnosis of a septic pulmonary embolus.

At this point (February 1984) the parents were prohibited from visiting the patient, and she was placed under constant observation (a nurse's aide was always at the bedside). A Broviac catheter was placed. Her body weight was $27 \cdot 2 \mathrm{~kg}$. She subsequently showed slow but progressive improvement. Her Broviac catheter was electively removed eight weeks later. (This was the first of eight central venous catheters to be electively removed.) She tolerated alimentation by gastrostomy tube and began to drink and eat. She was afebrile from midFebruary to the day of discharge (25 April). She was discharged to foster care weighing $36.7 \mathrm{~kg}$ after a total of 24 hospital admissions and 375 hospital days.

She has since returned to school and has not required admission to a hospital in the 15 months since discharge. Her weight is $40.8 \mathrm{~kg}$. The judge's ruling after a protracted trial in family court found the parents guilty of child abuse, and the child remains in foster care. 


\section{Discussion}

This child's gastrointestinal illness and bouts of recurrent infections continued for more than a year before her physicians' clinical impression that her illness was induced was strong enough to exclude her parents. The basis for suspecting that her infections were exogenously induced included: (1) Virtually all her infections were catheter associated; they were often polymicrobial or associated with organisms not usually associated with catheter associated sepsis. (2) Despite extensive radiologic investigations and surgical exploration (of abdomen and chest), no focus of infection or source for bloodstream seeding was identified. (3) A host defence problem was unlikely because extensive immunologic evaluation did not reveal appreciable abnormalities and the patient responded promptly to treatment and did not develop infections at sites commonly infected in patients with immunodeficiency (paranasal sinus, oral mucosa, lung, gastrointestinal tract, and skin other than catheter sites).

Similarly, the gastrointestinal ulceration remained inexplicable, with progressive ulceration while under optimal medical management. Multiple endoscopic procedures, blood tests, biopsy examinations and surgical explorations failed to find a cause. During admission to hospital there was one instance in which the mother reported bleeding from the gastrostomy tube, yet the red liquid recovered was guaiac negative. On another occasion, aspirin was detected in the blood, although it was never prescribed. Ingestion of a caustic substance or substances could have accounted for the gastrointestinal complaints as well as the endoscopic findings.

A pattern was discernable where the patient developed an exacerbation of gastrointestinal disease (often associated with new sites of ulceration) while at home and developed fever with bacteraemia in the hospital while an intravenous catheter was in place. The strongest evidence of exogenously induced illness was her remarkable recovery after exclusion of her parents and her good health during the 15 months since discharge while in foster care.

The patient's mother was probably responsible for inducing her illness. Her mother was at her bedside 24 hours a day during weekdays and seemed to be caring and attentive, features previously described in these mothers. ${ }^{5}$ She had a bland affect, avoided eye contact when conversing, was obese, and was noted by a consultant psychiatrist to be slightly depressed. Although she was not employed in the medical field, she showed medical sophistication. Contrary to the experience of Meadow, ${ }^{5}$ however, confrontation of this mother did not result in stopping of her child's infections and gastrointestinal symptoms. As our patient was often admitted from home with symptoms and had infections at three different hospitals it is inconceivable that medical personnel were involved in inducing her infections. Finally, we do not believe that the patient was responsible for inducing her own symptoms (Munchausen's syndrome). The patient was once heard asking her mother why she was fiddling with her intravenous line and when told of our suspicions she was incredulous. On one occasion, in which she developed a polymicrobial sepsis with infection at the catheter site, she could not have tampered with the catheter or tubing because she had spent the previous 24 hours with both hands restrained-one for the intravenous catheter and the other completely covered by a dressing for treatment of a previous thrombophlebitis. The child was interviewed on several occasions by a psychiatrist, social workers, and her primary physicians, but these discussions did not yield helpful information regarding the induction of her gastrointestinal illness or infections.

The severity of the symptoms prompted multiple invasive medical procedures. As without excluding the parents this child may have died ${ }^{15}$ it is critically important to consider the diagnosis of Meadow's syndrome in any child with inexplicable symptoms and signs, including recurrent episodes of polymicrobial septicaemia. ${ }^{34}$ Physicians must be cognisant of the increasing complexity of the presenting features of such cases as the presentation with both gastrointestinal disease and recurrent septicaemias led to delay in diagnosis. Among some of the physicians intimately involved in her care, there was disbelief and scepticism when legal measures were begun to exclude the parents. In the absence of an admission of guilt or clear cut proof of culpability a diagnostic (and therapeutic) trial of exclusion of the parent(s) may be necessary when this diagnosis is suspected.

\footnotetext{
References

${ }^{1}$ Meadow SR. Munchausen syndrome by proxy: the hinterland of child abuse. Lancet 1977;ii:343.

2 Rosen CL, Frost JD Jr, Bricker T, et al. Two siblings with recurrent cardiorespiratory arrest: Munchausen syndrome by proxy or child abuse? Pediatrics 1983;71:715-20.

3 Liston TE, Levine PL, Anderson C. Polymicrobial bacteremia due to polle syndrome: the child abuse variant of Munchausen by proxy. Pediatrics 1983;71:211-3.

${ }^{4}$ Halsey NA, Tucker TW, Redding J, et al. Recurrent noscomial polymicrobial sepsis secondary to child abuse. Lancet 1983;ii:558-60.

5 Meadow SR. Munchausen syndrome by proxy. Arch Dis Child 1982;57:92-8.
}

Correspondence to Dr L G Rubin, Schneider Children's Hospital of Long Island Jewish Medical Center, New Hyde Park, New York 11042, United States.

Received 25 March 1986 